Proceedings

\title{
A Study on the Perceptions of Autistic Adolescents towards Mainstream Emotion Recognition Technologies ${ }^{\dagger}$
}

\author{
Wendy Oude Nijeweme-d'Hollosy ${ }^{1, *}$, Tamara Notenboom ${ }^{1}$ and Oresti Banos ${ }^{1,2}$ \\ 1 BSS Telemedicine Group, Creative Technology, Faculty of Electrical Engineering, Mathematics and \\ Computer Science, University of Twente, 7522 NB Enschede, The Netherlands; \\ tamara.notenboom@gmail.com (T.N.); oresti@ugr.es (O.B.) \\ 2 Department of Computer Architecture \& Computer Technology, University of Granada, \\ E-18071 Granada, Spain \\ * Correspondence: w.dhollosy@utwente.nl; Tel.: +31-53-489-2766 \\ + Presented at the 12th International Conference on Ubiquitous Computing and Ambient Intelligence \\ (UCAmI 2018), Punta Cana, Dominican Republic, 4-7 December 2018.
}

Published: 23 October 2018

\begin{abstract}
Autistic people have difficulties in recognizing and expressing emotions from/to other people. Technologies can help to facilitate the communication and understanding between autistic and other people. This work particularly investigates the requirements autistic adolescents have on technologies that can measure bodily responses to recognize their emotions. A smartwatch, smartpatch and infrared camera were evaluated as potential everyday use devices to measure emotion. User requirements on emotion recognition technologies were elicited through an online survey (73 completed responses) and ten semi-structured interviews with autistic adolescents. The smartwatch is the preferred product, followed by the smart-patch. Infrared cameras are deemed unsuitable devices.
\end{abstract}

Keywords: autism; emotion recognition technology; smartwatch; smart-patch; infrared camera; human computer interaction (HCI); usability testing; sensor applications and deployments

\section{Introduction}

Autism, or autism spectrum disorder (ASD), is a neurodevelopmental disorder that is increasingly recognized. On average, it affects between $1 \%$ and $2 \%$ of people in the world with 4.5 times more males than females [1]. The symptoms differ per person but include weak social skills and weak communication skills reinforced by the inability to recognize emotions in oneself and in an interlocutor [2]. In this paper, we use the term "Autistic person" instead of "person with autism" based on the arguments described by Sinclair [3]. Not only the autistic person has troubles recognizing emotions in other people but also other people may have problems in recognizing emotions in the autistic person. This is because autistic people have different ways of conveying emotions [4].

Knowing the mental state of the other person plays an important role in social interactions. Not recognizing each other's emotions often leads to frustration on both sides. This is especially the case for autistic children, as it is also acknowledged by parents and teachers [5]. For autistic adolescents, miscommunication with peers complicates creating and maintaining relationships, often leading to a diminished social life of the autistic adolescent [6]. Therefore, this paper is especially focused on autistic adolescents in the age range 10 (puberty) to 18 (early adulthood) years old. There is an extensive list of possible interventions and training for autistic adolescents to improve social skills to 
ease the interaction with other people [7]. However, this approach expects an adaptation of the adolescent to its environment and it is still largely unknown to what extent training effects are translated into daily social life [8].

Instead of interventions to improve social skills, another approach to ease communication among autistic adolescents and others is the usage of emotion recognition technologies. Figure 1a shows the general way of how emotion recognition technology can be used during communication between two people. One person expresses an emotion ("Expresser"); the other person recognizes this emotion ("Recipient") by using emotion recognition technology.

Literature shows that innovative technology-based interventions for autism spectrum disorders are increasingly used for learning new social skills to people with ASD [7,9]. In general, these technology-based interventions can be expressed as the situation shown in Figure 1b: the autistic recipient has difficulty in perceiving the emotion of the expresser and the technology recognizes and translates the emotion of the expresser. This translation helps the autistic recipient to identify the expressed emotion with the intention to learn from this translation. Again, the approach in Figure $1 \mathrm{~b}$ expects an adaptation of the autistic recipient to its environment, after the autistic recipient has identified the expressed emotion.

In our research, we focus on the situation displayed in Figure 1c. Here, the autistic person is the expresser of an emotion and has no need to adapt to the environment. In this situation, the assumption is that the use of emotion recognition technology can help the recipient in perceiving the emotion of the autistic expresser. The development of an emotion recognition product that helps parents, teachers and peers to better understand the emotions of autistic children and adolescents is the target goal of our research.

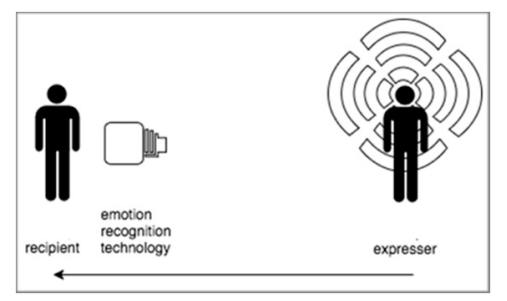

(a)

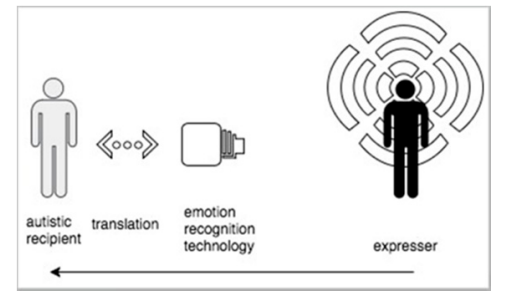

(b)

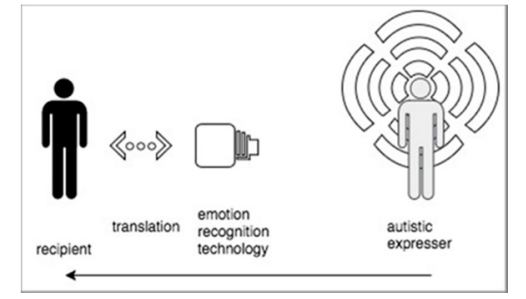

(c)

Figure 1. (a) Emotion recognition technology during communication between two people; (b) emotion recognition technology in innovative technology-based interventions for autism spectrum disorders; (c) Target situation of this research.

\section{Related Work}

Human emotions are expressed in many of our daily actions, and as such, they can be inferred in different ways, mostly via gesture, voice, facial expression, and neural and physiological signals $[9,10]$. Common used technologies can be used to measure human emotions, e.g., photo/video camera for facial expression and gesture, microphone for voice, EEG for neural signals, and ECG (skin electrodes), polyplethysmograph (pulse oximeter) and infrared camera for physiological signals.

Although emotion recognition technologies have been tested on human beings, these technologies have barely been argued, and proven, to work on autistic people. Especially the usage of facial, gesture and voice sensors on autistic people is complex, because every autistic person uses different and often reduced expressions [11]. For example, facial expressions by autistic people are often flat, neutral, robotic and peculiar [12]. Additionally, autistic children display reduced and weak movements for the muscles around the eyes and mouth [13]. The corners of the mouth are barely raised when autistic children smile, making the smile almost invisible [14]. Next to the flat expressions, both Czapinski and Bryson [13], and Yirmiya and others [12], detected atypical expressions that might account for the description of peculiar aspects.

In autistic people, emotion expressive body movement is often limited too. This is, because expressive gestures are lacking, and basic motor functions are impaired as shown in a rigorous metaanalysis on the subject of gait, postural balance, arm motor functions, and motor coordination [15]. 
Moreover, the whole posture is imbalanced both when standing still and in motion [16]. When standing still, autistic people had bigger balance sways and displayed repetitive movements for their centre of pressure [17]. Attwood and others demonstrated that autistic people did not convey expressive gestures that signify emotion in such cases [18].

Voice intonation in autistic people is also impaired, especially on emotional intonation. A lot of research has been done on the use of (non-emotional) intonation in autistic people where the prosodic qualities have been found dysfunctional. For example, autistic people are lacking in vocally expressing angriness, happiness, fear and especially sadness [19]. Next to this, also a higher pitch range was observed for emotional speech in autistic people [20].

This discordant convey of emotions is also described by the American Psychiatric Association's [21] description of expressing affect for autistic people: "marked impairments in the use of multiple nonverbal behaviors such as eye-to-eye gaze, facial expression, body postures, and gestures to regulate social interaction". Moreover, autistic persons can experience extreme overload internally, that is not visible externally; e.g., having a high heart rate, while outwardly appearing calm and relaxed [22]. Thus, physiological signals are a reaction on emotions that are fully functional in autistic people. For example, autistic people show normal skin response patterns to pleasant, unpleasant and neutral pictures [23]. Blood pressure and heart rate also show normal measurements in autistic people [24]. Even in case of alexithymia-i.e. pronounced difficulty in identifying and describing one's own emotions [25]-physiological responses did not seem to be influenced [23]. Therefore, physiological signals seem to be most suitable to measure emotions in autistic people, thus also in autistic adolescents. Next to this, the usage of physiological signals is also the most popular method of emotion monitoring [26].

\section{Study Objective}

Emotion recognition systems as displayed in Figure 1c should be accepted by the autistic expresser before these can actually be used. Therefore, user requirements have to be identified taking into consideration that autistic expressers can have a decreased capability to communicate, a high sensitivity to stimuli, a shallow area of interests, and restricted adaptability [2]. This paper describes the elicitation of the requirements of 10-18 year old autistic people on sensors that measure physiological signals for emotion recognition as the first step in the development of an emotion recognition system that can translate the emotions of autistic adolescents to their social environment.

\section{Methods}

We used a two-step qualitative research method to elicit the user requirements of autistic adolescents on technologies that can be used for emotion recognition. At first, an online survey was used to get a widespread picture on these requirements. This online survey was followed by interviews with ten autistic adolescents to allow for a more in-depth analysis.

\subsection{Choice of Emotion Recognition Technologies}

The focus of the questions in the online survey and interviews was on sensors that can measure physiological signals. From the state-of-the-art, it comes clear that different kinds of these sensors can be found in wearables such as smartwatches and wristbands. Next to this, physical signals can also be measured with (infrared) cameras. Therefore, the online survey focused on wearables as well as on cameras as most common emotion recognition technologies. In addition to these, a smart-patch was considered, as it could also be used for measuring relevant physiological signals that can be used for emotion recognition, as for example the HealthPatch ${ }^{\circledR} \mathrm{MD}$ from MediBioSense [27].

\subsection{Online Survey}

Asking autistic adolescents to fill in an online survey unsupervised is ethically problematic, because they are still children and extra vulnerable. Therefore, a proxy target group filled in the online survey on their behalf. The proxy target group consisted of two groups of people close to the 
original target group, namely group 1, non-autistic parents/caregivers of autistic adolescents that live in the same home as the parent/caregiver, and group 2, autistic adults (age $\geq 18$ ). Teachers, other family members and friends were not considered to be part of the proxy group as they might have not shared enough experiences with an autistic adolescent to answer all the questions.

The survey tool @SurveyMonkey was used to set up the online survey. In this survey, we questioned the participants on the following themes: (1) demographics (age and gender), (2) the way of recognition and expressing of emotions, (3) technologies that can be used to recognize emotions (wrist worn sensors, patches and cameras), (4) what aspects of these technologies may be found annoying, and (5) objects that may be bothersome to wear.

The participants had to fill in the online survey with the adolescent in mind, thus parents/caregivers about their child (proxy group 1) and autistic adults about their time as adolescents (proxy group 2).

Questions on the way of recognizing and expressing emotions could be answered through a 5point rating scale: never, almost never, sometimes, often, very often, with the added option "I don't know". Parents might not always know the child's attitude towards a question and may not be in the position to ask the child. This "I don't know" option prevented parents from guessing. Next to this, autistic people not always know themselves how they feel about a given problem.

The questions about technologies started with an explanation about physiological sensors and signals. Then the participants were asked if they were familiar with physiological sensors, followed by a list of possible kinds of objects that can be worn: (1) Bracelet, (2) Suspenders, (3) Glasses, (4) Watch, (5) Necklace, (6) Earrings, (7) Belt, and (8) Ring. From this list, the participants could select wearables that they found bothersome to wear (multiple selections possible) with the option to extend this list with their own answers. In addition, questions on wearing patches and watches were asked, and on the usages of cameras to monitor emotions.

To find participants for the online survey, an open URL was spread on online platforms. The platforms used were Facebook, a forum for autistic people [1], and the website of the Dutch organization for autism [28].

\subsection{Interviews}

The goal of the interviews was to test the reaction of the target group to several possible designs for emotion recognition technology. A proxy group would likely not have responded in the same way as the original target group, thus it was important to interview the actual target group.

The interviews were held with ten autistic adolescents. Inclusion criteria for the interviews were a diagnosis in the autistic spectrum, between 10 to 18 years old, average- or high-functioning, communicative, willing to participate, and parents/caregivers having given consent. In addition, some extra measures were taken. We made use of a familiar room to make the participant felt at ease and to ensure the participant was not busy processing the new environment. During each interview, a trusted adult was present, e.g., a parent or a teacher. This presence was intended to make the participant comfortable and ensure for their safety. In addition, printouts of the designs and coloring supplies were made available, so that the participant could draw instead of orally explain what they meant. The interview itself did not contain hypothetical questions, as autistic people often have trouble processing imaginary situations.

During the interviews, three examples of products that could be used as emotion recognition technology were presented: two wearables, a patch and an infrared camera. A microphone was used to record the interviews so that the interview could be transcribed afterwards. Before the interview, the participant and parent had to read the information pamphlet and to sign the consent form. The interviews took no longer than $30 \mathrm{~min}$ (excluding introduction and ending). The interview was semistructured. Next to the interview questions, age and gender were asked for statistical analysis.

The first wearable presented was the Gear S3 Frontier smartwatch from Samsung [29]. The smartwatch had a black design with a touchscreen, a rotating gear and two buttons. Optical sensors were visible on the back of the smartwatch. The sensors included an accelerometer, barometer, gyrosensor, heart rate monitor, ambient light sensor, GPS and microphone. The other wearable that was 
presented to the participants was the Angel Sensor M1 [30], an open-source wearable sensor that tracks heart rate, blood oxygen, skin temperature, steps, sleep quality, calories, acceleration, and orientation. This wearable did not have a screen as opposed to the smartwatch; it only sensed bio signals with optical sensors visible on the back of the wearable. The significantly different design of both wearables allowed the participants to compare and choose their favorite wearable. Next to the two wearables, also a patch was shown to the participants. This was the HealthPatch ${ }^{\circledR}$ MD from MediBioSense [27]. This patch could be placed on the chest using an adhesive layer. The patch contained a wearable physiological sensor that could measure ECG, heart rate, respiratory rate, skin temperature, body posture and number of taken steps. The patch could be placed somewhere on the body and this also gave insight into what location the participants would prefer when using a patch. The presented camera was the Kinect from Microsoft [31]. This is an infrared camera for tracking the body. The camera consisted of an infrared projector and camera that tracked the body and allowed $3 \mathrm{D}$ reconstruction. The Kinect also had an RGB camera, a depth sensor, and a microphone. The Kinect was put in a box that only showed the lenses, so that the participants did not immediately recognized it as a Kinect, because this could have influenced the experience and answers; the Kinect is already adopted for another purpose (gaming) and might therefore be felt familiar to the participants.

When a technology was presented during the interview, it was placed on the table. Next, the participant was encouraged to pick it up. In case a participant did not pick it up and started to talk about the product automatically, they were asked about their first thoughts about the product. Subsequently, the participant was asked if they felt comfortable about putting/turning the technology on and to motivate the answer to get more insight into the user requirements from the perspective of the participant. If the participant did not want to put the technology on, they were asked to explain why they did not want the device to be turned on. This explanation could give information on what aspects the device needed to improve on so that the participant would use it. Once the device was turned on, the participant was prompted to describe the experience and the aspects that they liked or disliked. Follow-up questions were asked when the answers remained too vague. Finally, participants were asked how they would stop the measurements if they fed up with these measurements. This question was asked to investigate whether the design of the technology was clear enough and to know whether the participant felt comfortable enough to operate the device.

Next to these open questions, the participant was also asked to give scores from 0 to 10 on four different topics for each presented device. 0 meant "totally not" and 10 meant "very much". The four topics were (1) the general likeability, (2) the feeling of control over the device, (3) the ease of use at home, and (4) the ease of use at school. The participant also got the opportunity to name, and draw, changes they would make on the shown technology. This was intended to reveal the personal preferences of the participant. It also helped pinpointing all the problems with the technology that the participant would like to see fixed before usage.

After all technologies were presented and discussed, additional questions on privacy issues were asked. As "who would be allowed to see your emotions?" and "at what moments are others allowed to see your emotions?" These questions were asked to get more information on the context of usage.

\subsection{Ethical Considerations}

The study was presented to the medical ethical committee (METC) Twente in Enschede, the Netherlands. We received a statement that ethics approval was not required for our study, as a normal healthcare path was not influenced by this study. Next to this, all participants, and in the interview study also their caregivers, had given informed consent before they were included.

\section{Results}

\subsection{Online Survey}

The survey was open for two weeks. During these weeks, we received 99 responses. Twenty-six responses were not completed and therefore filtered out, resulting into 73 complete responses. One 
of these 73 responses was excluded, because the respondent was a parent of a child younger than 10 years old. Subsequently, the final 72 responses were split up into two groups: (1) parents of autistic children between 10 and 18 years old, and (2) autistic adults $\geq 18$ years old (Table 1). Table 2 shows an overview of the responses to the online survey by the two groups.

Table 1. Demographics of the participants of the online survey.

\begin{tabular}{lll}
\hline \multicolumn{3}{c}{ Respondents $(\boldsymbol{n}=\mathbf{7 2})$} \\
\hline (1) Parents about their child (age 10-18) & (2) Autistic adult \\
\hline Number of responses & $18(25 \%)$ & $54(75 \%)$ \\
\hline \multirow{2}{*}{ Gender } & Male 14 $(78 \%)$ & $17(32 \%)$ \\
& Female 4 $(22 \%)$ & $37(69 \%)$ \\
\hline & 10 years 3 $(17 \%)$ & $18-24$ years $13(24 \%)$ \\
& 11 years 3 $(17 \%)$ & 25-29 years 5 $(9 \%)$ \\
& 12 years 2 $(11 \%)$ & $30-34$ years 3 $(6 \%)$ \\
Age & 13 years 3 $(17 \%)$ & $40-44$ years 8 $(15 \%)$ \\
& 14 years 1 $(6 \%)$ & $45-49$ years $9(17 \%)$ \\
& 15 years 0 $(0 \%)$ & $50-54$ years 5 $(9 \%)$ \\
& 16 years 1 $(6 \%)$ & $55-59$ years 2 $(4 \%)$ \\
& 17 years 5 $(28 \%)$ & $\geq 60$ years 3 $(6 \%)$ \\
\hline
\end{tabular}

Table 2. Overview of the responses to the online survey

\begin{tabular}{llll}
\hline Questions on Expressing Emotions & & & \\
\hline & Never & $0(0 \%)$ & $0(0 \%)$ \\
Q1. Do you ever have trouble expressing your & Almost never & $1(6 \%)$ & $3(6 \%)$ \\
emotions? & Sometimes & $3(17 \%)$ & $16(30 \%)$ \\
& Often & $10(56 \%)$ & $15(28 \%)$ \\
& Very often & $4(22 \%)$ & $19(35 \%)$ \\
& Don't know & $0(0 \%)$ & $1(2 \%)$ \\
\hline & Never & $0(0 \%)$ & $0(0 \%)$ \\
Q2. Do you ever have the feeling that others do not & Almost never & $1(6 \%)$ & $1(2 \%)$ \\
understand what you feel? & Sometimes & $0(0 \%)$ & $12(22 \%)$ \\
& Often & $9(50 \%)$ & $16(30 \%)$ \\
& Very often & $7(39 \%)$ & $24(44 \%)$ \\
& Don't know & $1(6 \%)$ & $1(6 \%)$ \\
\hline & Never & $0(0 \%)$ & $1(2 \%)$ \\
& Almost never & $1(6 \%)$ & $7(13 \%)$ \\
Q3. Are you ever confused about what you are feeling? & Sometimes & $5(28 \%)$ & $21(39 \%)$ \\
& Often & $5(28 \%)$ & $12(22 \%)$ \\
& Very often & $6(33 \%)$ & $12(22 \%)$ \\
& Don't know & $1(6 \%)$ & $1(2 \%)$ \\
\hline & Never & $0(0 \%)$ & $0(0 \%)$ \\
Q5. Would you like it if others would know what you & Almost never & $0(6 \%)$ & $2(4 \%)$ \\
& Almost never & $1(6 \%)$ & $2(4 \%)$ \\
Q4. Are there situations where you would like others & Sometimes & $5(28 \%)$ & $19(35 \%)$ \\
to know what you are feeling? & Often & $7(39 \%)$ & $16(30 \%)$ \\
& Very often & $4(22 \%)$ & $16(30 \%)$ \\
& Doften & $10(56 \%)$ & $20(37 \%)$ \\
\hline & & & \\
\hline
\end{tabular}




\begin{tabular}{llll}
\hline & Very often & $4(22 \%)$ & $15(28 \%)$ \\
& Don't know & $1(6 \%)$ & $2(4 \%)$ \\
\hline Questions on (Wearable) Technologies & & & \\
\hline \multirow{2}{*}{ Q6. Do you use an emotion communication technique? } & Yes & $1(6 \%)$ & $2(4 \%)$ \\
& No & $17(94 \%)$ & $52(96 \%)$ \\
& Don't know & $0(0 \%)$ & $0(0 \%)$ \\
\hline Q7. Have you ever worn a physiological sensor & Yes & $1(6 \%)$ & $8(15 \%)$ \\
(biosensor)? & No & $17(94 \%)$ & $46(85 \%)$ \\
& Don't know & $0(0 \%)$ & $0(0 \%)$ \\
\hline \multirow{2}{*}{ Q8. Is wearing a watch bothersome? } & Yes & $8(44 \%)$ & $16(30 \%)$ \\
& No & $10(56 \%)$ & $38(70 \%)$ \\
& Don't know & $0(0 \%)$ & $0(0 \%)$ \\
\hline \multirow{2}{*}{ Q9. Is wearing a patch bothersome for you? } & Yes & $5(28 \%)$ & $15(28 \%)$ \\
& No & $10(56 \%)$ & $35(65 \%)$ \\
\hline \multirow{2}{*}{ Q10. Would wearing a patch be less bothersome when } & Don't know & $3(17 \%)$ & $3(6 \%)$ \\
there is a nice picture on the patch? & Yes & $1(6 \%)$ & $5(9 \%)$ \\
& No & $14(78 \%)$ & $45(83 \%)$ \\
Q11. Is being photographed bothersome for you? & Don't know & $3(17 \%)$ & $4(7 \%)$ \\
\hline & Yes & $10(56 \%)$ & $27(50 \%)$ \\
& No & $7(39 \%)$ & $22(41 \%)$ \\
Q12. Is being filmed bothersome for you? & Don't know & $1(6 \%)$ & $5(9 \%)$ \\
\hline
\end{tabular}

In the survey, participants could also select the following objects that were found bothersome to wear: bracelet, suspenders, glasses, watch, necklace, earrings, belt, and ring. Multiple selections were possible, with the option to extend this list with other objects. The analysis on these data was made via word clouds, were the size of the words denoted the frequency of each element labelled as being bothersome.

Figure 2a shows this word cloud for autistic children as noticed by their parents (group 1). Figure $2 \mathrm{~b}$ shows this word cloud for autistic adults (group 2). The objects designated by the respondents were divided into three groups: tightly worn objects, dangling objects and objects with a specific texture. Objects with a specific texture (itchy, inflexible, denim, clothing labels, wool, plastic) were not predefined, but respondents added these themselves to the list. In most cases, both children and adults had trouble to give clear and concise explanations why wearing these objects is annoying to them. When a clear motivation was given, that motivation could usually be led back to a sensory overload, which is a common problem in autism [2].

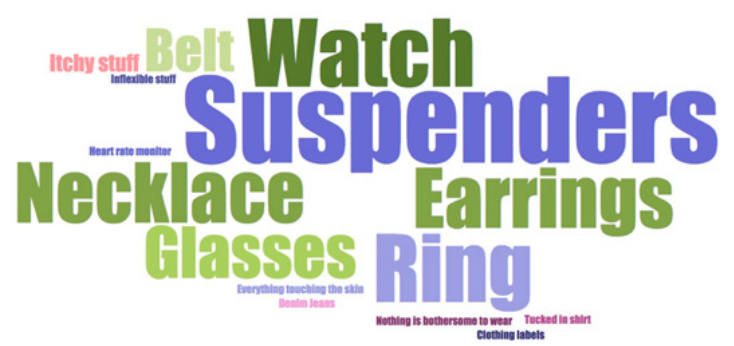

(a)

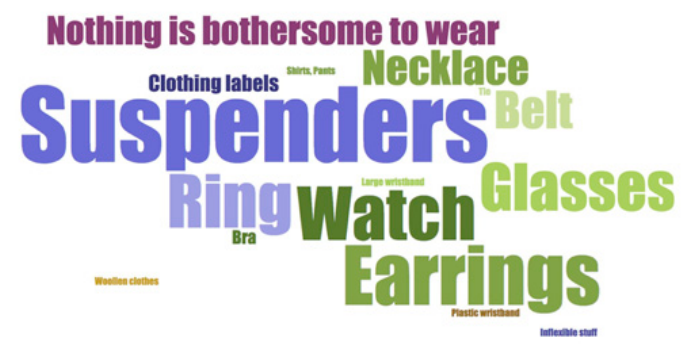

(b)

Figure 2. Objects that are bothersome to wear for (a) autistic children as noticed by their parents (group 1), and (b) for autistic adults (group 2). 


\subsection{Interviews}

The interviews were conducted with 10 autistic teenagers between 10 and 18 years old that were gathered from different schools and families. All the participants finished the interview, so no selection was required because of incomplete responses. Sixty percent of the participants were male and $40 \%$ were female. The mean age of the group was 14 years old $(\mathrm{SD}=1.76)$.

\subsubsection{Wearable on the Wrist}

All of the participants were willing to wear the Samsung S3 Gear smartwatch and turned it on. They were curious about the functions and did not need any encouragement to pick it up and turn it on. Both the boys and the girls expressed the wish that the design was less black. The boys redesigned the smartwatch with black, grey, dark blue and dark green while the girls chose a combination of bright blue and pink/purple. The smartwatch was too heavy for the children and they redesigned it smaller and lighter. Many participants also had trouble taking the smartwatch on and off and disliked the material of the band. Two participants had a leather allergy. The Angel M1 wearable was not preferred as wearable on the wrist, even though it was smaller and lighter. The reason for this was that the M1 wearable does not have a touchscreen and it is not multifunctional. Next to this, the sensors on the back of the Angel M1 were raised a bit, which felt irritating to some of the participants. One participant preferred a wearable on the ankle instead of the wrist.

The smartwatch was endorsed with a good average general likeability $(7.67 / 10)$. The feeling of control on the smartwatch was sufficient for most of the participants (6.65/10), although two participants graded lower than 5 for the feeling of control. Some participants suggested redesigning the smartwatch with an on/off button for the measurements solely. All participants were convinced that an on/off button should be red so that it can be recognized immediately. A signal that indicates that the device is measuring was also discussed, but this should not be a light since the participants found lights distracting. The smartwatch received higher scores for usage at school (7.44/10) than home (7.05/10).

\subsubsection{Health Patch}

Most participants, except one, were willing to wear the health patch. They were more hesitant to pick it up than the smartwatch, and described the patch as "medical", "scary" and "weird". They also found the patch smaller than they expected, with the exception of one participant that fantasized about the patch being much bigger with chips, wires, and LEDs like a bionic arm. The participants were asked where they would place the patch. The upper and lower arm were most frequently chosen. One participant would place it on the back of the torso and another would place it on the stomach. The patch should originally be placed over the heart, which was acceptable for all the participants, although they were more inclined towards their own chosen spots. The participants barely felt the patch on their skin, except for a pulling sensation when moving the body part the patch was placed on, and when taking the patch off.

The patch was also up for redesign by the participants. They immediately started with changing the surface to a beige skin colour, "like a normal band aid" and so that it "would not be as obvious". The raised middle of the patch with the chip was also disapproved; it should be flat. However, there should be a button included to turn the measurements on or off. A display, or indicating light, was not encouraged by the participants because this would look out of place on a patch and could be distracting. Two participants also mentioned that electrodes were visible through the cover and that this should be prevented.

The patch scored a relatively high general likeability (7.42/10) and an insufficient grade on the feeling of control (4.65/10). Usage at home scored higher (6.84/10) than usage at school (4.65/10). Usage at school use was not valued high because of the medical design of the health patch, defined as scary and weird by most participants. Next to this, they were insecure about what their peers would say about wearing a health patch. 


\subsubsection{Infrared Camera}

Eighty percent of the participants already reported feeling stared at before the camera was even turned on. The interest in the infrared camera was low but turning it on was allowed by all of the participants. They reported that the blinking light on the front of the camera was annoying. They also thought the camera was too big, and four participants suggested a webcam-sized infrared camera mounted in a corner on the wall.

Turning the infrared camera off posed a small problem. The participants needed more time to think about this and answered hesitantly. The solution they came up with was to pull the power plug, or step out of the sight, of the infrared camera, neither of which they found convenient. One participant suggested a remote control for the infrared camera, and then quickly had the idea that this could be done with a smartphone app. The other participants wanted to place an on/off button on the camera itself, and again, this button should be red.

The infrared camera scored low on all the elements. The general likeability was barely sufficient (5.7/10). The other factors were insufficient. Usage at school scored lower (3.55/10) than usage at home (4.9/10), because the participants did not think it was practical to have to drag it with them to every room, plug it into a power source and set it so that they are in the view of the infrared camera. At home, this is also unpractical but still more feasible to them. The control was also below sufficient $(4.9 / 10)$.

\subsubsection{Preferred Product}

The smartwatch was preferred as usable product for $70 \%$ of the interview participants. The health patch was preferred by $30 \%$ of the participants. The infrared camera was preferred by no one and it also had the least suggestions that would change this preference. The patch might come closer to first place if the suggestions they made for redesign were incorporated.

\subsubsection{Privacy}

The interviewed participants liked the idea that their emotions could be measured to inform the environment about their emotions, and that the data can be viewed by themselves and others, as (grand)parents, teachers, brothers, sisters and friends. Multiple participants voiced interest in getting to know themselves better. Ninety percent of the participants would allow their parents to view their emotions; one participant would not allow anyone other than himself to see the emotions. Eighty percent of the participants wanted to be able to decide for themselves when someone could see their emotions, depending on time and place. This is the reason why a clear on/off button is essential. One participant mentioned that instead of an on/off button, a pause button might also be an option. In that case, measurements would stop for a predefined period of time (e.g., $30 \mathrm{~min}$ ).

\section{Discussion}

Related work showed that physiological signals seem to be most suitable to measure emotions in autistic people. Therefore, the online survey and interviews in our study were focused on technologies that can measure physiological signals. This study resulted into relevant information on the attitude towards, and requirements on, emotion recognition technologies from the perspective of autistic adolescents.

The first three questions in the online survey on expression emotions showed that autistic adolescents often experience trouble with expressing emotions and therefore very often have the feeling that others do not understand what they feel. They are also sometimes confused about their own feelings, although autistic children are more often confused about their own feelings (61\%) than autistic adults (44\%). This might imply that autistic adults may be more skilled in recognizing their own emotions because of having more life experiences. These results of the online survey correspond to what is found in literature [2,22].

The online survey also showed that both autistic children (61\%) and autistic adults $(60 \%)$ wish that others would know what they are feeling in specific situations. The interviews also showed that 
autistic adolescents would like it when others can see their emotions in specific situations. Therefore, it can be concluded that an emotion recognition system that can translate the emotions of autistic adolescents to their social environment will be a helpful tool for this target group.

Both the online survey and the interviews elicited information that could be used to set up guidelines for the design of a system that translates emotions of an autistic adolescent (expresser) to another person-e.g., parent, teacher, peer-(recipient). These guidelines are:

- Use physiological signals, because these signals are trustworthy to monitor emotions in autistic people. Other signals such as facial expressions, body language and voice intonation are employed differently by autistic people and are therefore not reliable to translate the emotions of an autistic adolescent to others.

- A multifunctional smartwatch design around the wrist would be best to monitor emotions based on physiological signals. A health patch would be the second choice. Autistic adolescents generally dislike the usage of (infrared) cameras.

- There should be a clearly recognizable on/off button to start or halt the monitoring of emotions in order to give the expresser control over the device.

- The design should be familiar to a product known by the expresser; e.g., a normal watch or a skin-coloured band-aid.

- The design should not be dangling, not too tight, and as flexible as possible; a smartwatch band should have different length possibilities and a health patch should be flexible enough not to pull the skin during regular or abrupt movements.

- The design should be smaller and lighter than the Gear S3 Frontier smartwatch [29] and the HealthPatch from Medibiosense [27].

- Textures are important because of the sensitivity of the skin of autistic people [2]. For the smartwatch, a natural band of smooth texture is advisable. Leather could be possible. Metal or ceramics might be an option because of the smoothness and the thermal properties of this material that keep it at skin temperature.

- For all used materials, possible allergies of the expresser should be taken into account.

- Putting a product on, and taking it off, should be as simple and painless as possible for the expresser.

- $\quad$ Surfaces have to be flat, so no raised bumps or protruding buttons.

- An indication that shows monitoring is on/off is preferred, but not in the form of (blinking) lights that draw attention and distracts the expresser.

- Avoid a medical-looking design by hiding electrodes from view and not making the design predominantly white.

- Make inputs and outputs available to both the expresser as the recipient, for example through a smartphone app.

- A recipient should be accepted or certified by the expresser to allow viewing the emotions of the latter.

This work has some limitations. In the online survey, gender distribution was uneven over the two groups of participants (Group 1: Parents about their autistic child (10-18), Group 2: Autistic adults). The autistic children were overrepresented by males $(78 \%)$ and the autistic adults were overrepresented by females $(68 \%)$. It would have seemed more likely that males would be overrepresented in both groups, since ASD is 4.5 times more prominent in males [1].

The study described in this paper has led a set of guidelines for the design of a system that translates emotions of an autistic adolescent to be interpreted by another person. These guidelines are based on information elicited from a group of average/high-functioning, communicative, and willing to participate autistic participants. Therefore, one should still be aware that within the autistic spectrum there are a lot of individual differences and personal preferences that should be considered when using the proposed guidelines. 


\section{Conclusions and Future Work}

This work has intended to shed light on how autistic children perceive modern emotion recognition technologies, which represent a potential solution to support communication in between (non)autistic people but could also negatively impact their daily living. This work constitutes the foundation for a more ambitious goal, which is to develop a system that is able to translate emotions of an autistic adolescent to another person, especially to support parents and teachers in the communication with their autistic child and pupil, but also to help the autistic adolescent in the communication with their peers. However, a secondary objective can be added, and that is the support of the self-learning process of one's emotions, because this study also showed that there is confusion in autistic people about their own emotions. The next step in this research will be the development of a prototype system, based on the results of this study and the described guidelines. This prototype will be used in experimental tests on accuracy, efficiency, usability and benefits as the next step in the development of an emotion recognition system that can translate the emotions of autistic adolescents to their social environment.

Author Contributions: Conceptualization, W.O.N.H., T.N. and O.B.; Methodology, W.O.N.H., T.N. and O.B.; Formal Analysis, T.N.; Investigation, T.N.; Resources, T.N. and W.O.N.H.; Data Curation, T.N. and W.O.N.H..; Writing-Original Draft Preparation, W.O.N.H. and T.N.; Writing-Review \& Editing, O.B. and W.O.N.H.; Visualization, T.N., W.O.N.H. and O.B.; Supervision, O.B. and W.O.N.H.; Project Administration, O.B., W.O.N.H. and T.N.; Funding Acquisition, O.B.

Funding: This research was partly funded by the research project "Progress in Computer Architectures for Automatic Learning using Heterogeneous Sources: Health and Well-Being Applications" (TIN2015-71873-R).

Acknowledgments: The authors would kindly like to thank the participants in the online survey and interviews for their time, effort, and willingness to cooperate in our research.

Conflicts of Interest: The authors declare no conflict of interest.

\section{References}

1. Data and Statistics | Autism Spectrum Disorder (ASD) I NCBDDD | CDC. Available online: https://www.cdc.gov/ncbddd/autism/data.html (accessed on 31 May 2018).

2. Milton, D. So what exactly is autism? AET Competence framework for the Department for Education. 2012. Available online: http://www. aettraininghubs. org. uk/wp-content/uploads/2012/08/1_So-what-exactly-isautism. Pdf (accessed on 31 May 2018).

3. Sinclair, J. Why I dislike "person first" language. Autonomy, the Critical Journal of Interdisciplinary Autism Studies. 2013. Available online: http://www.larry-arnold.net/Autonomy/index.php/autonomy/ article/view/OP1/html_1 (accessed on 17 October 2018).

4. Samson, A.C.; Huber, O.; Gross, J.J. Emotion regulation in Asperger's syndrome and high-functioning autism. Emotion 2012, 12, 659, doi:10.1037/a0027975.

5. Bearss, K.; Johnson, C.; Smith, T.; Lecavalier, L.; Swiezy, N.; Aman, M.; McAdam, D.B.; Butter, E.; Stillitano, C.; Minshawi, N.; et al. Effect of parent training vs parent education on behavioral problems in children with autism spectrum disorder: A randomized clinical trial. JAMA 2015, 313, 1524-1533, doi:10.1001/jama.2015.3150.

6. Orsmond, G.I.; Krauss, M.W.; Seltzer, M.M. Peer relationships and social and recreational activities among adolescents and adults with autism. J. Autism Dev. Disord. 2004, 34, 245-256, doi:10.1023/B:JADD.0000029547.96610.df.

7. Grynszpan, O.; Weiss, P.L.; Perez-Diaz, F.; Gal, E. Innovative technology-based interventions for autism spectrum disorders: A meta-analysis. Autism 2014, 18, 346-361, doi:10.1177/1362361313476767.

8. Berggren, S.; Fletcher-Watson, S.; Milenkovic, N.; Marschik, P.B.; Bölte, S.; Jonsson, U. Emotion recognition training in autism spectrum disorder: A systematic review of challenges related to generalizability. Dev. Neurorehabil.2018, 21, 141-154, doi:10.1080/17518423.2017.1305004.

9. Sharmin, M.; Hossain, M.M.; Saha, A.; Das, M.; Maxwell, M.; Ahmed, S. From Research to Practice: Informing the Design of Autism Support Smart Technology. In Proceedings of the $2018 \mathrm{CHI}$ Conference on Human Factors in Computing Systems, Montréal, QC, Canada, 21-26 April 2018; p. 102. 
10. Jerritta, S.; Murugappan, M.; Nagarajan, R.; Wan, K. Physiological signals based human emotion recognition: A review. In Signal Processing and its Applications (CSPA). In Proceedings of the IEEE 7th International Colloquium, Penang, Malaysia, 4-6 March 2011; pp. 410-415.

11. Sherer, M.R.; Schreibman, L. Individual behavioral profiles and predictors of treatment effectiveness for children with autism. J. Consult. Clin. Psychol. 2005, 73, 525, doi:10.1037/0022-006X.73.3.525.

12. Yirmiya, N.; Kasari, C.; Sigman, M.; Mundy, P. Facial expressions of affect in autistic, mentally retarded and normal children. J. Child Psychol. Psychiatry 1989, 30, 725-735, doi:10.1111/j.1469-7610.1989.tb00785.x.

13. Czapinski, P.; Bryson, S.E. Reduced facial muscle movements in Autism: Evidence for dysfunction in the neuromuscular pathway? Brain Cogn. 2003, 51, 177-179.

14. Loveland, K.A.; Tunali-Kotoski, B.; Pearson, D.A.; Brelsford, K.A.; Ortegon, J.; Chen, R. Imitation and expression of facial affect in autism. Dev. Psychopathol. 1994, 6, 433-444, doi:10.1017/S0954579400006039.

15. Fournier, K.A.; Hass, C.J.; Naik, S.K.; Lodha, N.; Cauraugh, J.H. Motor coordination in autism spectrum disorders: A synthesis and meta-analysis. J. Autism Dev. Disord. 2010, 40, 1227-1240, doi:10.1007/s10803010-0981-3.

16. Fournier, K.A.; Kimberg, C.I.; Radonovich, K.J.; Tillman, M.D.; Chow, J.W.; Lewis, M.H.; Hass, C.J. Decreased static and dynamic postural control in children with autism spectrum disorders. Gait Posture 2010, 32, 6-9, doi:10.1016/j.gaitpost.2010.02.007.

17. Fournier, K.A.; Amano, S.; Radonovich, K.J.; Bleser, T.M.; Hass, C.J. Decreased dynamical complexity during quiet stance in children with autism spectrum disorders. Gait Posture 2014, 39, 420-423, doi:10.1016/j.gaitpost.2013.08.016.

18. Attwood, A.; Frith, U.; Hermelin, B. The understanding and use of interpersonal gestures by autistic and Down's syndrome children. J. Autism Dev. Disord. 1988, 18, 241-257, doi:10.1007/BF02211950.

19. Macdonald, H.; Rutter, M.; Howlin, P.; Rios, P.; Conteur, A.L.; Evered, C.; Folstein, S. Recognition and expression of emotional cues by autistic and normal adults. J. Child Psychol. Psychiatry 1989, 30, 865-877, doi:10.1111/j.1469-7610.1989.tb00288.x.

20. Nadig, A.; Shaw, H. Acoustic and perceptual measurement of expressive prosody in high-functioning autism: Increased pitch range and what it means to listeners. J. Autism Dev. Disord. 2012, 42, 499-511, doi:10.1007/s10803-011-1264-3.

21. Edition, F. Diagnostic and Statistical Manual of Mental Disorders; American Psychiatric Publishing: Arlington, VA, USA, 2013.

22. Picard, R.W. Future affective technology for autism and emotion communication. Philos. Trans. R. Soc. B Biol. Sci. 2009, 364, 3575-3584, doi:10.1098/rstb.2009.0143.

23. Allen, R.; Davis, R.; Hill, E. The effects of autism and alexithymia on physiological and verbal responsiveness to music. J. Autism Dev. Disord. 2013, 43, 432-444, doi:10.1007/s10803-012-1587-8.

24. Bölte, S.; Feineis-Matthews, S.; Poustka, F. Brief report: Emotional processing in high-functioning autismphysiological reactivity and affective report. J. Autism Dev. Disord. 2008, 38, 776-781.

25. Griffin, C.; Lombardo, M.V.; Auyeung, B. Alexithymia in children with and without autism spectrum disorders. Autism Res. 2016, 9, 773-780, doi:10.1002/aur.1569.

26. Okada, G.; Yonezawa, T.; Kurita, K.; Tsumura, N. Monitoring Emotion by Remote Measurement of Physiological Signals Using an RGB Camera. ITE Trans. Media Technol. Appl. 2018, 6, 131-137, doi:10.3169/mta.6.131.

27. HealthPatch ${ }^{\circledR}$ MD-MediBioSense. Available online: http://www.medibiosense.com/products/healthpatch/ (accessed on 31 May 2018).

28. NVA-Actuele Oproepen. Available online: http://www.autisme.nl/prikbord/deelnemers-aan-onderzoekgezocht/actuele-oproepen.aspx (accessed on 31 May 2018).

29. Gear S3 Frontier 46 mm Smartwatch (Bluetooth) Dark Gray. Available online: https://www.samsung.com/ us/mobile/wearables/smartwatches/samsung-gear-s3-frontier-sm-r760ndaaxar/ (accessed on 31 May 2018).

30. Angel Sensor M1-AngelList. Available online: https://angel.co/projects/170164-angel-sensor-m1?src= startup_profile (accessed on 31 May 2018).

31. Microsoft Xbox 360 Kinect Review: Microsoft Xbox 360 Kinect-CNET. Available online: https://www.cnet. com/uk/products/microsoft-xbox-360-kinect/review/ (accessed on 31 May 2018).

(C) 2018 by the authors. Licensee MDPI, Basel, Switzerland. This article is an open access article distributed under the terms and conditions of the Creative Commons Attribution (CC BY) license (http://creativecommons.org/licenses/by/4.0/). 